\section{Langzeitüberleben bei Glioblastom}

Der Anteil von Glioblastompatienten, die noch länger als zwei Jahre nach Diagnosestellung leben, nimmt mit der Verbesserung der Therapieprotokolle stetig zu. Experten der renommierten Mayo Clinic gingen deshalb der Frage nach, welche prognostischen Faktoren bei diesen Patienten auf eine lange Überlebenszeit hinweisen.

n die Analyse gingen die Daten von 10.022 Gliompatienten aus der SEER (Surveillance Epidemiology and End Results)-Datenbank ein. Bei allen war zwischen 1998 und 2008 ein Glioblastom diagnostiziert worden. Die Arbeitsguppe um Derek R. Johnson wertete u. a. für verschiedene Zeitintervalle bedingte Überlebenswahrscheinlichkeiten aus, d.h. die Wahrscheinlichkeit für eine definierte Zeit am Leben zu bleiben - unter der Bedingung, dass die Patienten bereits zuvor ein bestimmtes Zeitintervall nach Diagnosestellung überlebt hatten.

Die mediane Überlebenszeit der gesamten Population lag bei 12,6 Monaten. Ein Jahr nach der Diagnosestellung lebten noch 4.684 Patienten, nach zwei Jah- ren 1.474, 198 nach fünf Jahren; acht Patienten lebten noch nach zehn Jahren.

Mit zunehmend längerer Überlebenszeit nach Diagnose vergrößerte sich auch die weitere Überlebenswahrscheinlichkeit. So errechneten die Autoren beispielsweise eine 2-Jahres-Überlebenswahrscheinlichkeit von 19,8\% für Patienten, bei denen die Diagnose unmittelbar zuvor gestellt worden war. Hatten die Patienten zwei Jahre nach der Diagnosestellung überlebt, so lag die Wahrscheinlichkeit, zwei weitere Jahre zu überleben, bei $38,1 \%$. Waren die Patienten sogar fünf Jahre nach der Diagnosestellung am Leben, errechnete sich eine Überlebenswahrscheinlichkeit für die nächsten zwei Jahre von 65,9\%.
Faktoren, die mit einer höheren Mortalität einhergingen, waren in der univariaten Analyse höheres Alter (bis zu 6,4-faches Risiko bei über 80-Jährigen), männliches Geschlecht, Status als Lediger, Diagnosestellung in den Jahren 1998-2004, asiatische Herkunft und keine Totalresektion des Tumors. Bei der multivariaten Analyse zeigte sich nur noch das höhere Alter als prognostisch ungünstiger Faktor, der durchgehend unmittelbar nach Diagnose, nach einem Jahr sowie drei Jahre nach der Diagnosestellung mit einer höheren Sterbewahrscheinlichkeit assoziiert war.

Fazit: Die vorliegende Studie zeigt, dass eine möglichst lange Überlebenszeit nach Diagnose eines Glioblastoms auch die Chance auf ein weiteres Überleben deutlich vergrößert. Hohes Alter erwies sich in allen Analysen als schlechter prognostischer Faktor.

Andreas Fischer

Johnson DR et al. Conditional probability of long-term survival in glioblastoma. A population based analysis. Cancer. 2012;118(22): 5608-13.

\title{
Ältere Glioblastom-Patienten: Faktoren für ein längeres Überleben
}

\section{Die meisten Überlebensdaten für das Glioblastom stammen von Studienteilnehmern, die bei Diagnose zwischen 18 und 70 Jahre alt waren. Ein US-ameri- kanisch-französisches Team hat nun Prognosefaktoren für über 70-jährige Patienten errechnet.}

$\mathrm{D}$ ie Forscher um Jacob G. Scott schlossen in ihre Auswertung Patienten ein, bei denen im Alter von $\geq 70$ Jahren ein Glioblastom diagnostiziert worden war. Sie prüften mithilfe der rekursiven Partitionierungsanalyse eine Vielzahl von möglichen Risikofaktoren. Bei diesem Verfahren werden verschiedene dichotome Merkmale (z. B. Alter über oder unter 75 Jahre; Operation oder nur Biopsie) eingesetzt.

Das Ziel besteht darin, die Variablen so zu wählen, dass Patientengruppen resultieren, die sich in einem Zielparame- ter (z.B. „Überlebenszeit“) deutlich unterscheiden.

Die Methode führten die Autoren zunächst bei 437 Patienten aus zwei Studien durch. Das Ergebnis war ein Modell, das vier Gruppen mit verschiedenen Überlebenszeiten ergab. Patienten der ersten Gruppe mit einem medianen Überleben von 9,3 Monaten waren im Alter von unter 75,5 Jahren diagnostiziert worden, und bei ihnen war eine partielle oder großräumige Resektion erfolgt. Gruppe 2 bestand aus Patienten über 75,5 Jahren, bei denen ebenfalls eine Resektion des Tumors erfolgt war (medianes Überleben: 6,4 Monate). Patienten der dritten Gruppe mit einem Karnofsky-Index von 70-100\% ohne Tumorresektion, nur mit Biopsie, überlebten 4,6 Monate. In Gruppe 4 befanden sich Patienten, ebenfalls ohne Resektion, die einen Karnofsky-Index von unter $70 \%$ aufwiesen; ihr medianes Überleben lag bei 2,3 Monaten.
In einem zweiten Schritt wendeten Scott und Kollegen die Einteilung nach diesen vier Gruppen bei 265 Patienten der dritten, einer französischen, Studie an. Sie konnten dabei signifikant unterschiedliche Überlebenszeiten nachweisen, die für die vier Gruppierungen 8,5, 7,7, 4,3 bzw. 3,1 Monate betrugen ( $\mathrm{p}<$ $0,001)$.

Fazit: In dieser Studie wurden mithilfe der rekursiven Partitionierungsanalyse vier verschiedene Gruppen älterer Glioblastompatienten definiert, die sich in ihrer Lebenserwartung signifikant unterschieden. Die Arbeitsgruppe sieht großes Potenzial in diesem statistischen Modell, das sich auch für andere Variablen wie etwa Tumormarker eignen könnte.

Andreas Fischer

Scott JG et al. Recursive partitioning analysis of prognostic factors for glioblastoma patients aged 70 years or older. Cancer. 2012;118(22): 5595-600. 